\title{
Comparison of off-axis TMA and FMA telescopes optimized over different fields of view: applications to Earth observation
}

\author{
L. Clermont* ${ }^{\mathrm{a}}$, Y. Stockman ${ }^{\mathrm{a}}$, W. Dierckx ${ }^{\mathrm{b}}$, J. Loicq $^{\text {a }}$ \\ ${ }^{\mathrm{a} C e n t r e ~ S p a t i a l ~ d e ~ L i e ̀ g e, ~ U n i v e r s i t y ~ o f ~ L i e ̀ g e, ~ A v e n u e ~ d u ~ P r e ́ ~ A i l y, ~ L i e ̀ g e, ~ B e l g i u m ; ~}$ \\ ${ }^{\mathrm{b}}$ VITO, Boereatang 200, Mol, Belgium
}

\begin{abstract}
TMA, or three mirror anastigmats, have already been used successfully for various space missions. In the frame of earth observation, ProbaV satellite uses 3 TMAs to cover a total $102.4^{\circ}$ field-of-view; ground sampling distance is about $100 \mathrm{~m}$ at the center of field-of view and $370 \mathrm{~m}$ at the edge. For future earth observation missions, the goal would be to reach $100 \mathrm{~m}$ spatial resolution all over the $102.4^{\circ} \mathrm{FOV}$. This would require to up-scale optical specifications, thus increasing geometrical aberrations. FMA, or four mirror anastigmats, could thus be a good candidate for future missions, as a fourth mirror would allow better correction of optical aberrations. In this work, TMA and FMA have been optimized over different fields-of view. Performance limitations are then derived, which show that FMA seems promising for future missions. Radiometry aspects are discussed and preliminary tolerance analysis is carried out.
\end{abstract}

Keywords: TMA, FMA, optical design, earth observation, space instrument, ProbaV, vegetation

\section{INTRODUCTION}

\subsection{Background: TMA and their applications to earth observation}

Off-axis TMAs, or three mirror anastigmats, are widely used for applications such as space and earth observation. They indeed provide high image quality over large fields-of-view. Because such systems are purely reflective, they are achromatic and can operate over a wide spectral domain. Moreover, TMAs made of material such as aluminum are lightweight and thus suit well for space missions. In addition, when both mirrors and mount are manufactured of the same material (ex: aluminum), temperature variations in space induce the same dilatation for every elements and thus optical properties are scaled. While off-axis TMA are advantageous due to the absence of obscuration, the main drawbacks concern alignment issues as well as stray-light.

Off-axis TMAs provide wide-field observation across the instrument symmetry plane [1]. Along the symmetry plane, the field is very limited (Figure 1). On a push broom configuration, where the wide-field is oriented across-track, 2D scenes are recorded thanks to satellite motion. In multispectral imagers, each pixel line is covered by a spectral filter: during satellite motion, the same scene is recorded at a different wavelength and with a small time-offset. In this case, the widefield is called the spatial field and the small field is called the spectral field. For a matter of simplicity, spatial field will simply be called field-of-view (FOV).
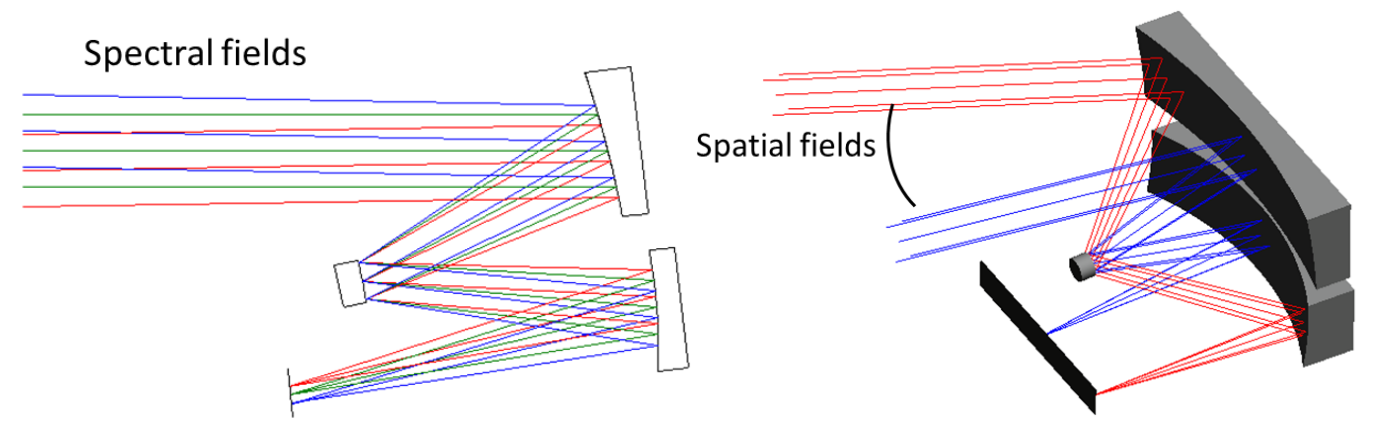

Figure 1 - In a TMA, spatial field is perpendicular to the plane of symmetry of the system; spectral field is in the plane of symmetry. *lionel.clermont@ulg.ac.be; phone +32 43824696; csl.ulg.ac.be 
In the frame of earth observation, TMA have already been successfully used on missions such as ProbaV [2][3] or Advanced Land Imager (ALI) [4]. In the specific case of ProbaV, the mission aim is to provide daily monitoring of vegetation reflectivity all over the Earth. The mission goal is in the continuity of SPOT-Vegetation missions [3]. The data are used for applications such as monitoring the spread of deserts and deforestation. On ProbaV three TMAs, with individual field-of-view of $34^{\circ}$, are used in a fan-like configuration to cover a total $102.4^{\circ}$ field of view. The use of multiple TMAs to cover the full FOV is necessary due to optical quality degradation for a too large FOV. On a heliosynchrone orbit at $820 \mathrm{~km}$ from earth, it provides daily images of the earth with spatial resolution of about 100 meters at the center of FOV, 370 meters at the edge (VNIR). The difference between the ground sampling distance (GSD), at the center of FOV and edge of FOV, comes from the earth curvature and the fact that angular resolution (iFOV) is constant. As the graph on Figure 2 shows, the GSD increases fast with the field-of-view. Increasing slightly the FOV might require a significant increase of instrument resolution if we want to keep the same edge GSD.

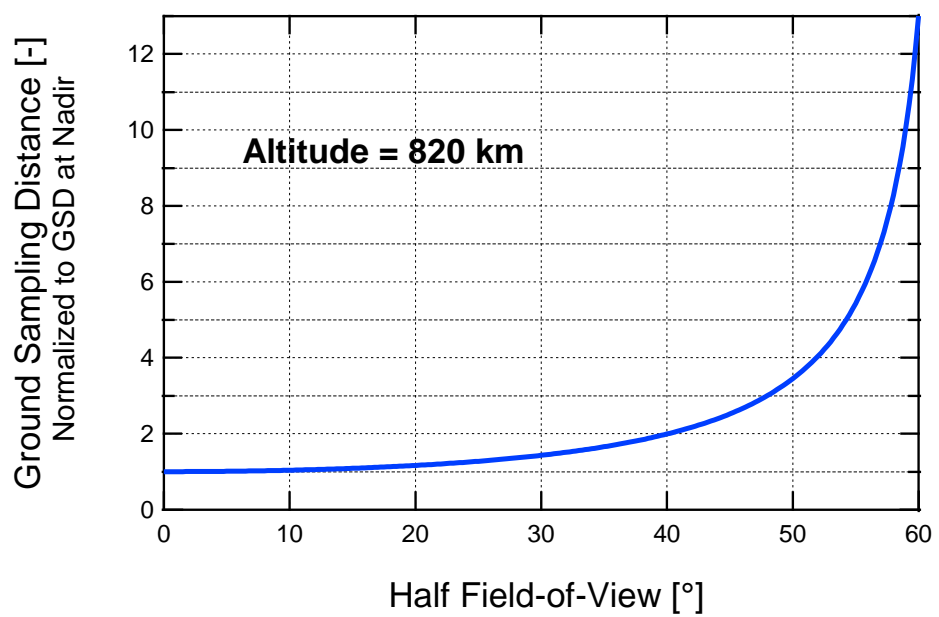

Figure 2 - Evolution of GSD with respect to half FOV for an instrument at $820 \mathrm{~km}$ from the scene. GSD is normalized to its value at Nadir (i.e FOV $=0^{\circ}$ )

In the case of ProbaV, spectral bands go from visible and near-infrared (VNIR) to short-wavelength infrared (SWIR). The bands are defined by central wavelength $\lambda$ and spectral width $\Delta \lambda$ (Table below). All the bands are imaged with the same instrument (same focal length). A folding mirror is used to deviate the image plane for some of the spectral field, in order to use a specific detector for VNIR and another one for SWIR. SWIR pixels are twice the size of VNIR pixels; consequently GSD is twice broader for SWIR than for VNIR.

Table 1 - Spectral range of ProbaV

\begin{tabular}{ccc}
\hline \hline Band & $\boldsymbol{\lambda}(\mathbf{n m})$ & $\boldsymbol{\Delta} \boldsymbol{\lambda}(\mathbf{n m})$ \\
\hline Blue & 464 & 45 \\
Red & 665 & 80 \\
IR & 843 & 143 \\
SWIR & 1600 & 70 \\
\hline \hline
\end{tabular}

\subsection{Limitations of TMA for future earth observation missions: FMA as an alternative}

For a successor mission of earth observation, there is a need of increasing the ground sampling distance while keeping the daily coverage. This is indeed the conclusion that has been made from a survey at the intention of worldwide users of earth observation data [5]. Increasing the ground sampling distance can be obtained by two ways: either keeping the same payload while decreasing the orbit altitude of the satellite, or by increasing the instrument resolution. Decreasing the orbit altitude is not relevant; GSD is indeed proportional to the altitude, but the required orbit altitude will be 
unstable during time. Moreover, an altitude reduction would decrease the swath thus affecting daily coverage. Improvement of the resolution is thus compulsory.

Increasing the resolution requires to increase the instrument size, consequently increasing the geometrical aberrations. This would result in a decrease of image quality, especially at the edge of the individual instruments field-of-view. Compared to ProbaV, more than 3 TMAs with a smaller individual field would thus be required. This is why the use of FMA, or four mirror anastigmats, could bring added values. Indeed, for large fields-of-view, FMA are supposed to provide better image quality than TMA. This comes from the fact that the fourth mirror allows a better correction of the optical aberrations. At identical focal length and pupil size, thus at identical resolution, a larger field-of-view could be achieved with acceptable optical quality for FMA than for TMA. The use of FMA rather than TMA would consequently minimize the total number of instruments required. The main disadvantages of FMA concerns alignment and tolerance issues.

\subsection{Objective}

The goal of this work is to investigate the capabilities of TMAs and FMAs, regarding the requirements of the successor earth observation mission. Ground sampling distance should be increased significantly, while keeping the same total FOV and without changing the orbit altitude (giving a swath of about $2250 \mathrm{~km}$ ). In particular, the goal is to get a ground sampling distance of about $100 \mathrm{~m}$ all over the 102.4 ${ }^{\circ}$ FOV. TMAs and FMAs are optimized over different fields-ofview. Performance limitations are derived. They allow concluding about the possibility to reach the goal.

\section{METHODS}

\subsection{System specifications definition}

Spatial resolution $\left(\mathrm{x}_{\mathrm{res}}\right)$ is determined by altitude $\left(\mathrm{H}_{\text {sat }}\right)$ and curvature of the Earth, but also by the focal length (f) of the optical system and the detector pitch $\left(\mathrm{x}_{\text {pitch }}\right)$. The spatial resolution at the nadir-point can be derived from the following formula:

$$
\mathrm{x}_{\mathrm{res}}(\mathrm{m})=\mathrm{H}_{\mathrm{sat}}(\mathrm{km}) \frac{x_{\text {pitch }}(\mu m)}{f(m m)}
$$

For deriving the spatial-resolution at the edge of the FOV, a more complicated formula is required which takes into account the curvature of the Earth and the FOV. With the approximation of a spherical Earth with radius $\mathrm{R}_{\text {earth }}$ $6378.135 \mathrm{~km}$, the following formula applies:

$$
\mathrm{x}_{\mathrm{res}}(\mathrm{m})=\frac{x_{\text {pitch }}(\mu m)}{f(m m)}\left(\left(R_{\text {earth }}+H_{\text {sat }}\right) \frac{\cos (F O V / 2)}{\sqrt{1-\left(\frac{R_{\text {earth }}+H_{\text {sat }}}{R_{\text {earth }}} \sin (F O V / 2)\right)^{2}}}-R_{\text {earth }}\right)
$$

With $\mathrm{H}_{\mathrm{sat}}=820 \mathrm{~km}$, pitch of $13 \mu \mathrm{m}$ (pixel size) and focal length of $110 \mathrm{~mm}$, the spatial resolution at-nadir is $97 \mathrm{~m}$. For a field-of-view of $102.4^{\circ}$, the spatial resolution is then $366 \mathrm{~m}$ at the edge, thus with a ratio of 3.7 with respect to the nadirvalue (Figure 2).

Thanks to these formulas, the required focal length can be derived. At altitude of $820 \mathrm{~km}$, for a $100 \mathrm{~m}$ GSD at the edge of FOV, the required focal length is $385 \mathrm{~mm}$. In this case, GSD at the center of FOV would be $27 \mathrm{~m}$. Without optical aberration aspects, the image is adequately sampled on the detector if the diffraction pattern (Airy disk) is less or equal to the detector-pixel. With this consideration, the pupil diameter $\mathrm{D}$ is (with $\lambda$ for wavelength): 


$$
\mathrm{D} \geq 2 \times \frac{1.22 \times \lambda \times f}{x_{\text {pitch }}}
$$

As the formula is wavelength dependant and the spectral domain is going from VNIR to SWIR, a compromise must be chosen. For example, the graphs on Figure 3 show the evolution of the minimal pupil size as a function of the wavelength. Two cases are presented, 100m GSD and 27m GSD at the center of FOV. The second case corresponds to a $100 \mathrm{~m}$ GSD at the edge of FOV. Both VNIR and SWIR detectors are illustrated. In these specific cases, a pupil size of respectively $15.5 \mathrm{~mm}$ and $55.5 \mathrm{~mm}$ are good compromises.
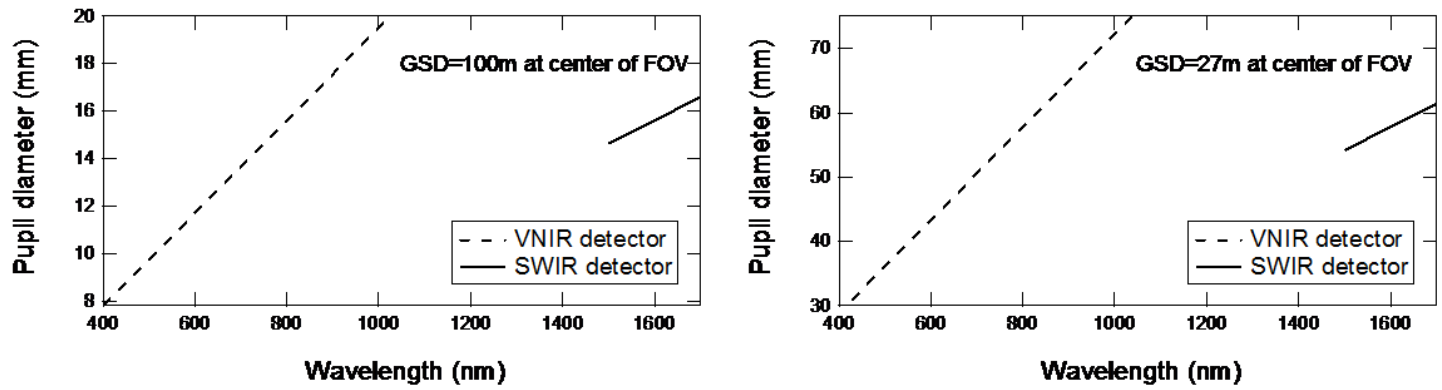

Figure 3 - Pupil diameter D to sample the Airy disk on a pixel. Case of VNIR detector (Pix=13 $\mu \mathrm{m})$ and SWIR detector $(\mathrm{Pix}=26 \mu \mathrm{m})$ : On the left, the GSD at center of FOV is $100 \mathrm{~m}$, which gives a pupil of $15.5 \mathrm{~mm}$. On the right, GSD at the center of FOV is $27 \mathrm{~m}$, which gives a pupil diameter of $55.5 \mathrm{~mm}$.

\subsection{Optical design}

Taking as a baseline the values of focal length and pupil diameter derived from Equation 2 and Equation 3, instruments are designed using the $\mathrm{CodeV}$ software. The instruments are optimized over a spectral field of $\pm 1^{\circ}$ and over different spatial fields.

The optimization was made in several steps, with an optimization on the spot size (error function). The "Global Synthesis" algorithm was first used to explore error function topology and generate a bunch of different instrument configurations. Some of these configurations were then used as a baseline for more complete optimizations. The best result, in terms of MTF, was then selected. MTF is evaluated at Nyquist frequency, which only depends on the pixel size:

$$
\text { Nyquist }=\frac{1}{2 \times x_{\text {pitch }}}
$$

The baseline assumption is that pixel pitch is $13 \mu \mathrm{m}$ in VNIR and $26 \mu \mathrm{m}$ in SWIR. Thus, Nyquist frequency is $38.46 \mathrm{lp} / \mathrm{mm}$ for VNIR and $19.23 \mathrm{lp} / \mathrm{mm}$ for SWIR. In the first step of the optical design process, no tolerances were taken into account.

During optimization process, tilts and decentering of mirrors and detector were allowed. Several constraints were applied on the system, including realistic spacing between the elements. For example, ray-clearance has to be controlled for avoiding shadowing [6]. The aperture stop is placed on the secondary mirror, which gives a primary and tertiary mirror with reasonable sizes. In the case of TMA, we constrain the shape of the primary mirror to be aspheric. The secondary mirror is spherical and the tertiary mirror is conic. This choice was made for staying in continuity with ProbaV. In the case of FMA, the fourth mirror is chosen aspheric.

From PROBA-V case analysis, we concluded that in order to fulfill the MTF requirements with tolerances, the system without tolerances should have an MTF above 0.6 at Nyquist. Consequently, by hypotheses it is considered that the instruments fulfill requirements if the system without tolerances gives an MTF of at least 0.6. Preliminary tolerance analysis is performed for validating this hypothesis. 
Different cases are analyzed. First, optimization is performed for TMA with optical specifications similar as for ProbaV $(\mathrm{f}=110 \mathrm{~mm}, \mathrm{D}=15,5 \mathrm{~mm})$, GSD is therefore about $100 \mathrm{~m}$ at the center of FOV. TMA are then optimized with up-scaled optical specifications $(\mathrm{f}=365 \mathrm{~mm}, \mathrm{D}=55.5 \mathrm{~mm})$; GSD is $27 \mathrm{~m}$ at the center of FOV, $100 \mathrm{~m}$ at the edge of FOV. With these same up-scaled specifications, optimization is then performed on FMA.

\subsection{Size and weight evaluation}

Size and weight are crucial points for space missions. Comparison of the weight is performed based on calculation of the weight of the mirrors only, considering these are made of aluminum. Mounts are not considered in the weight estimation; indeed the aim is not to give accurate values but to compare the different configurations. Size is evaluated considering the volume of the instruments envelope (cube), when these are placed in the typical fan-like configuration. Again, this is not accurate and is made for comparison only.

\section{RESULTS AND DISCUSSION}

\subsection{TMAs with $\mathbf{f}=\mathbf{1 1 0} \mathrm{mm}, \mathrm{D}=\mathbf{1 5 . 5} \mathrm{mm}$}

TMAs with $110 \mathrm{~mm}$ focal length and $15.5 \mathrm{~mm}$ pupil diameter were optimized over different fields-of-view. With such specifications, GSD is around $100 \mathrm{~m}$ at the center of FOV (altitude of $820 \mathrm{~km}$ ). The graph on Figure 4(a) shows the minimum MTF of the TMAs, at Nyquist frequency, as a function of the field-of-view on which they have been optimized. MTF are plotted at the extreme wavelengths of both VNIR and SWIR bands.
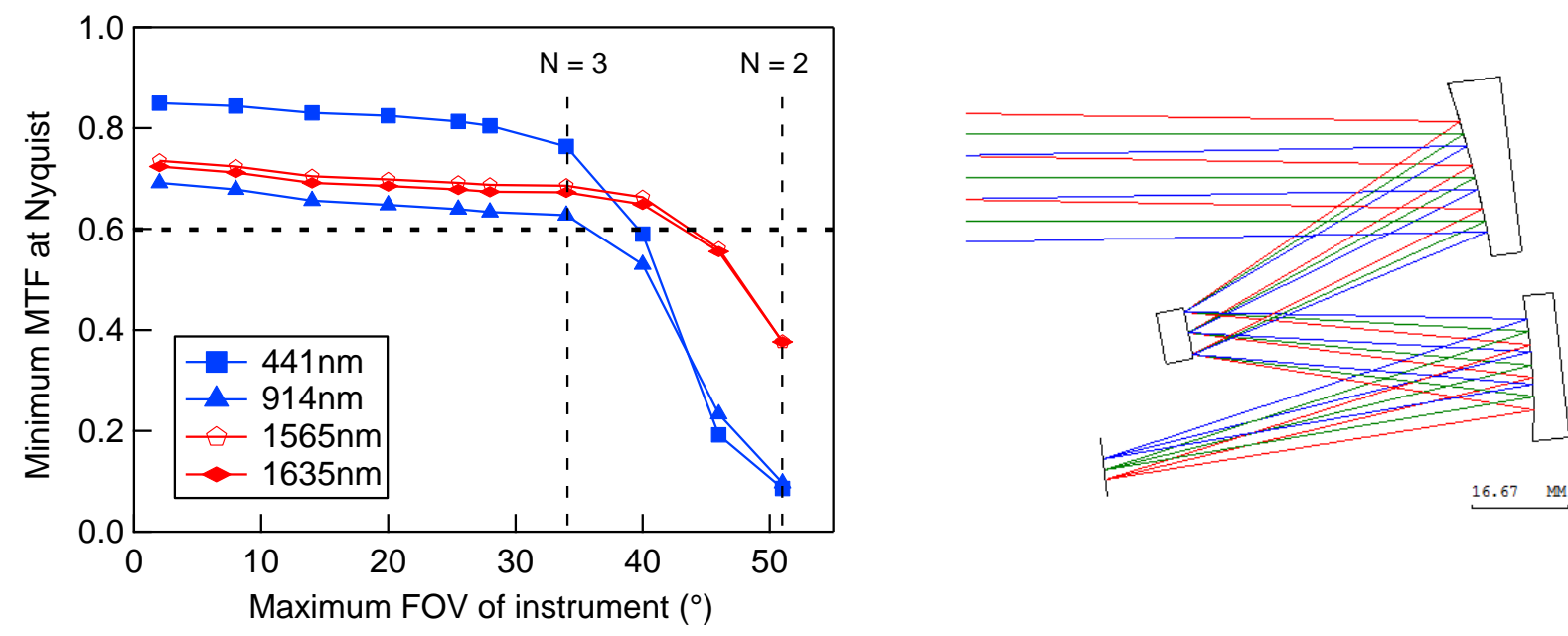

Figure 4 - (a) Minimum value of MTF of the camera, at Nyquist frequency, as a function of the maximum FOV of each camera. N corresponds to the number of TMAs used for covering the entire FOV. Tolerances are not taken into consideration. (b) TMA for N=3.

MTF decreases when the optimization FOV increases, moreover MTF is smaller for higher wavelengths. MTF should be worst in SWIR than in VNIR; however this is not the case as, due to pixel size, Nyquist frequency is twice smaller for SWIR than VNIR. The graph shows that TMA fulfill MTF specifications only for a field-of-view $\leq 36^{\circ}$. Indeed, by hypothesis the required MTF is 0.6 because tolerances were not taken into account. Consequently, for covering the full $102.4^{\circ}$ field-of-view, at least 3 TMAs are required. This is indeed the case for ProbaV.

In the case where 3 TMAs are used for covering the full field-of-view, the weight of the mirrors for one TMA is $126 \mathrm{~g}$. For the three TMAs, the mirrors weight is $378 \mathrm{~g}$. The envelope of one TMA corresponds to $0.5 \mathrm{dm}^{3}$, when arranged in a fan-like configuration the overall 3 TMAs envelope has a volume of $3 \mathrm{dm}^{3}$. Distortion gives a maximum spectral field deviation of $0.23^{\circ}$ and a maximum spatial field deviation of $0.26^{\circ}$. In VNIR, the number of pixels required for one camera is 4747 pix/band. The three cameras thus require 14242 pix/band. 


\subsection{TMAs with $\mathrm{f}=385 \mathrm{~mm}, \mathrm{D}=55.5 \mathrm{~mm}$}

Analogous optimization has been performed on TMAs with up-scaled optical specifications. In this case, GSD is $27 \mathrm{~m}$ at the center of FOV. Because specifications are up-scaled while pixel size stays the same, geometrical aberrations increase and optical quality thus decreases. This is indeed what is observed on the graph on Figure 5(a): degradation is small for small FOV but is significant for large FOV. Due to the increase of geometrical aberrations, MTF remains high enough (0.6) only until a field-of-view of about $21^{\circ}$.
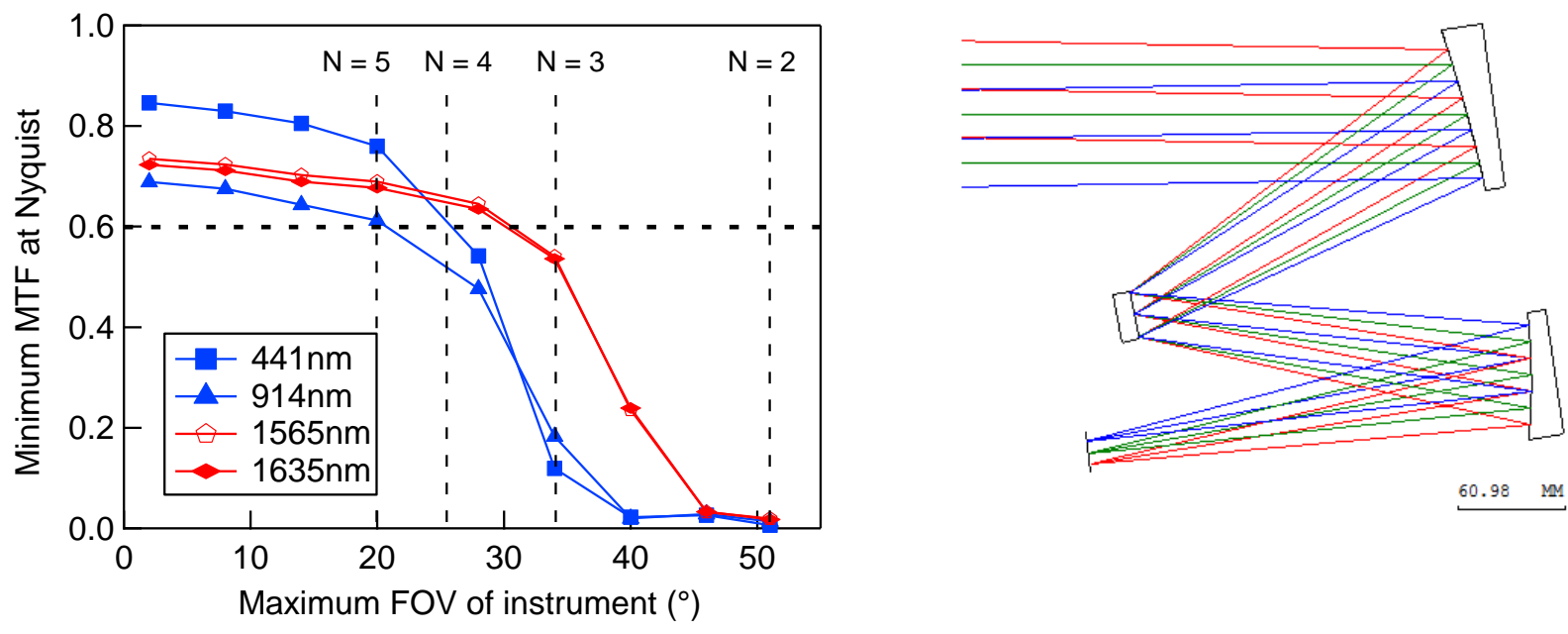

Figure 5 - (a) Minimum value of the MTF of the camera, at Nyquist frequency, as a function of maximum FOV of each camera. N corresponds to the number of TMAs used for covering the entire FOV. Tolerances are not taken into consideration. (b) TMA for N=5.

As a consequence, at least 5 TMAs are required for covering the full field-of-view. In this case, the individual FOV would be $20.4^{\circ}$; the weight of the mirrors of 1 TMA is then $2 \mathrm{~kg}$. The total mirrors weight for the 5 TMAs would therefore be $10 \mathrm{~kg}$. Even considering mirrors which are lightened, which would give a mass reduction of about $70 \%$, the mirror weight is still very heavy for space missions. The envelope of one TMA has a volume of $14 \mathrm{dm}^{3}$, the 5 TMAs envelope is $165 \mathrm{dm}^{3}$. Distortion is reasonable, with $0.03^{\circ}$ maximum spectral field deviation and $0.13^{\circ}$ maximum spatial field deviation. In VNIR, the number of pixels required for one camera is $10550 \mathrm{pix} / \mathrm{band}$. The five cameras thus require 52748 pix/band, 3.7 times more than for ProbaV specifications.

\subsection{FMAs with $\mathrm{f}=385 \mathrm{~mm}, \mathrm{D}=55.5 \mathrm{~mm}$}

In this case, optical specifications are the same as previously (GSD $=27 \mathrm{~m}$ at the center of FOV), but the instruments that are designed are FMAs. As shown in Figure 6(a), for FMA, MTF stays high until a much higher field-of-view than for TMA of same optical specifications. MTF requirements are fulfilled until fields-of-view above $50^{\circ}$. Consequently, at least two instruments are required for covering the full swath. 

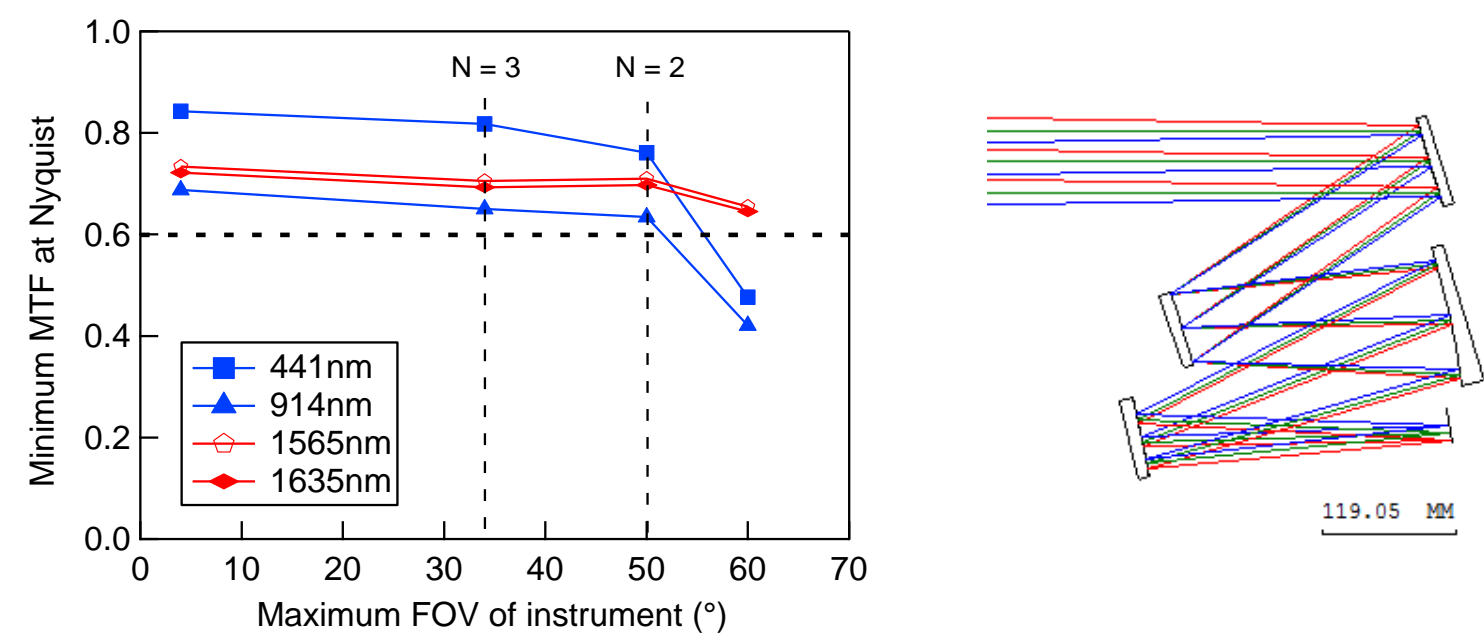

Figure 6 - (a) Minimum value of MTF of the camera, at Nyquist frequency, as a function of the maximum FOV of each camera. N corresponds to the number of FMAs used for covering the entire FOV. Tolerances are not taken into consideration. (b) FMA for N=2.

The mirrors of an FMA with FOV of $50^{\circ}$ have a weight of $2.1 \mathrm{~kg}$; The mirrors of the two FMA, needed for covering the total field-of-view, thus have a weight of $4.2 \mathrm{~kg}$. This is less than half the weights of previous case in which 5 TMAs are used. Individual FMA envelope has a volume of $30 \mathrm{dm}^{3}$, the total instrument envelope volume is $120 \mathrm{dm}^{3}$. On the other hand, as a drawback the distortion is significantly larger than for TMA, maximum spectral field deviation is $2.08^{\circ}$ and maximum spatial field deviation is $1.02^{\circ}$. Moreover, it is suspected that FMAs are less tolerant than TMAs and thus for FOV as large as $50^{\circ}$ the image quality could in practice be much smaller. The number of pixels required is identical to the case of scenario 1 .

Three FMAs with individual FOV of $34^{\circ}$ could be used for covering the full swath. In this case, distortion is smaller; it gives $0.66^{\circ}$ maximum spectral field deviation and $0.19^{\circ}$ maximum spatial field deviation. The weight of the mirrors of each FMA is $1.69 \mathrm{~kg}$; the total 3 FMAs system is heavier than for two FMAs of $50^{\circ} \mathrm{FOV}$. Instead of using 3 identical FMAs, nearly 100m GSD could be obtained by replacing the central camera by a ProbaV-like TMA. The central TMA would provide GSD of 100m at the center of its FOV, 110m at the edge of its FOV; FMAs on the edges would provide GSD of maximum $100 \mathrm{~m}$. This solution would reduce the weight and size of the payload. Moreover, the total number of pixels would be reduced due to the smaller resolution of central camera.

\section{RADIOMETRY}

Signal-to-noise ratio (SNR) is a critical aspect that has to be analyzed. We are interested here on the impact on SNR of using either TMA or FMA. The SNR writes:

$$
\mathrm{SNR}=\frac{\operatorname{Rad} \cdot \delta \lambda \cdot A \cdot \cos (\theta) \cdot T \cdot \tan (i F O V)^{2} \cdot \frac{\lambda}{h \cdot c} Q E \cdot t_{i}}{\text { noise }}
$$

With $\mathrm{Rad}=$ radiance $\left(\mathrm{W} / \mathrm{m}^{2} / \mathrm{st} / \mu \mathrm{m}\right) ; \delta \lambda=\operatorname{spectral}$ width $(\mu \mathrm{m}) ; \mathrm{A}=$ pupil diameter; $\mathrm{T}=$ system transmission $=\mathrm{T}_{\text {Filter }} \times \mathrm{T}_{\mathrm{Optics}} ;$ $\mathrm{QE}=$ quantum efficiency; $\mathrm{t}_{\mathrm{i}}=$ integration time $(\mathrm{s}) ; \mathrm{iFOV}=\operatorname{atan}(\mathrm{GSD} / \mathrm{h})$ the angular resolution. The signal is proportional to $\mathrm{A} \cdot \cos (\Theta)$, which is the pupil projected along the normal to the light direction. Because of this factor the SNR depends on the field: the relative signal between a scene observed with one camera at the center of FOV and at the edge of its FOV is $\cos \left(\mathrm{FOV}_{\text {camera }} / 2\right)$. 
The fact of considering either TMA or FMA would impact on SNR due to imperfect reflectivity of mirrors. Indeed, with aluminum for example, reflectivity of mirror is about $98 \%$ (Vis). TMA thus has an optical transmission of $94 \%$, while for FMA it would be $92 \%$. Filters transmission could be increased slightly to compensate for the reflectivity losses. Consequently, at equal optical specifications, SNR for FMA is significantly lower than for TMA only when field-ofview is significantly larger. When comparing TMA of $34^{\circ} \mathrm{FOV}$ and FMA with $50^{\circ} \mathrm{FOV}$, for example, the signal difference is $7 \%$.

\section{TOLERANCE ANALYSIS}

\subsection{Methods}

Tolerance analysis is performed assuming a theoretical alignment of the system: both alignment errors and manufacturing errors are taken into account. The principal idea of the calculation is to extract some orders of magnitude of MTF degradation when tolerances are taken into account. The analysis is first performed on TMA with FOV of $34^{\circ}$, $\mathrm{f}=110 \mathrm{~mm}$ and $\mathrm{D}=15.5 \mathrm{~mm}$. Tolerance analysis is then performed on FMA with FOV of $34^{\circ}, \mathrm{f}=385 \mathrm{~mm}$ and $\mathrm{D}=55 . \mathrm{mm}$. The tolerance parameters and values are the following:

Table 2 - Tolerance parameters

\begin{tabular}{lc}
\hline \hline Translation of the mirrors and of the detector along X, Y and Z (local axis) & $0.03 \mathrm{~mm}$ \\
Rotation of the mirrors and of the detector around X, Y and Z (local axis) & 1 \\
Tolerance on radius of curvature & $1 \%$ \\
Tolerance on conic constant & $0.10 \%$ \\
Tolerance on first order aspheric parameter & $0.10 \%$ \\
Tolerance on second order aspheric parameter & $1 \%$ \\
\hline \hline
\end{tabular}

These values might not be perfectly accurate; however it allows making preliminary tolerance analysis. The merit function is MTF at the Nyquist frequency. Tolerance analysis is performed at the highest wavelength in VNIR $(914.5 \mathrm{~nm})$, this is indeed the wavelength at which MTF is the worst.

\subsection{Tolerance analysis of TMA}

In this case, tolerance analysis is performed considering two compensators: shift along optical axis of mirror 2 and shift along optical axis of the detector. The result is that, at $3 \sigma$, MTF at Nyquist stays above 0.41 (Figure 7).

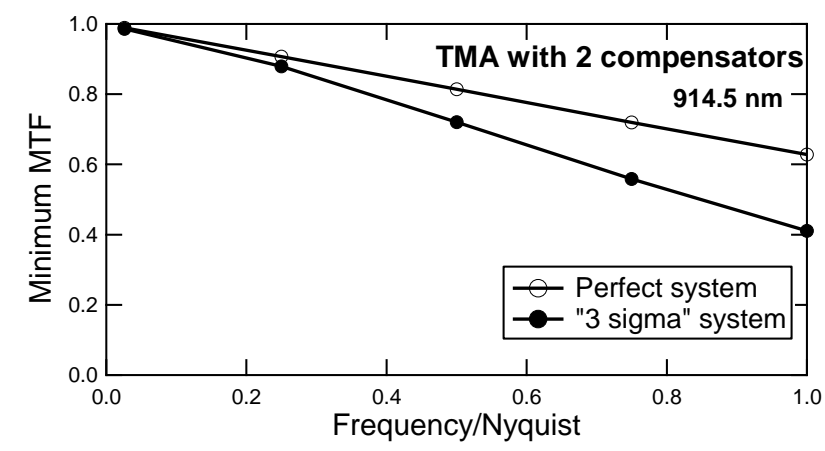

Figure 7 - Minimum MTF for TMA $\left(\mathrm{FOV}=34^{\circ}, \mathrm{f}=110 \mathrm{~mm}, \mathrm{D}=15.5 \mathrm{~mm}\right)$ in perfect case and with tolerance at $3 \sigma$. Two compensators are considered. 


\subsection{Tolerance analysis of FMA}

First, tolerance analysis is made with the same compensators as in TMA case: shift along optical axis of mirror 2 and shift along optical axis of the detector. In this case (Figure 8 (a)) the decrease of MTF at Nyquist goes down to 0 at $3 \sigma$. Specifications are clearly not fulfilled. In order to limit performance degradation, an extra compensator must be introduced. For example, the extra compensator can be the tilt of mirror 1 . In this case (Figure 8 (b)), at $3 \sigma$ MTF at Nyquist stays above 0.37 .
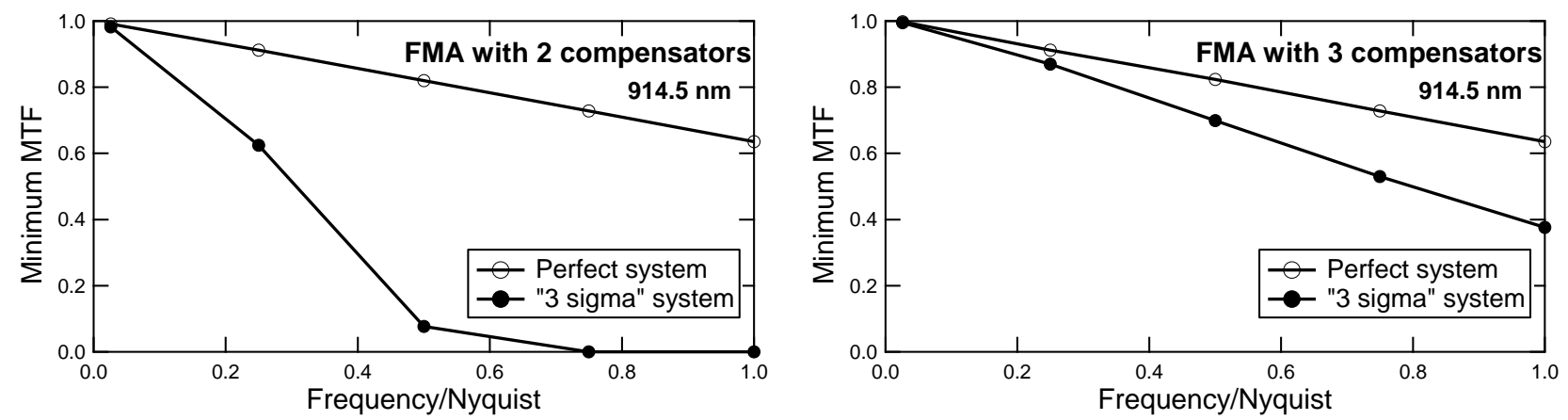

Figure 8 - Minimum MTF for FMA (FOV=34 $4^{\circ}, \mathrm{f}=385 \mathrm{~mm}, \mathrm{D}=55.5 \mathrm{~mm}$ ) in perfect case and with tolerance at $3 \sigma$. (a) Two compensators. (b) Three compensators.

\section{CONCLUSIONS}

TMAs and FMAs have been optimized over different fields-of-view. Specifications similar to ProbaV have been considered. Specifications have then been up-scaled in order to reach $100 \mathrm{~m}$ GSD all over the $102.4^{\circ}$ FOV. Results show that, for ProvaV specifications, at least 3 TMAs are required for covering a full $102.4^{\circ}$ FOV. For up-scaled specifications, geometrical aberrations are up-scaled and at least 5 TMAs are required. FMAs provide high image quality over a larger field than TMA, at least 2 FMAs are required for covering the $102.4^{\circ} \mathrm{FOV}$. In terms of radiometry, TMA and FMA of identical FOV give nearly the same SNR. Significant decrease of SNR only arises when considering instruments with much larger FOV. Preliminary tolerance analysis has been performed. It has been shown that FMA requires 3 compensators while TMA requires 2 . The main conclusion of these calculations is that FMA seems very promising for a future earth observation mission. However, the tolerance analysis has to be completed in further work for confirming these results.

\section{Acknoledgements}

This work was supported in the frame of Prodex contract C4000107501; the authors are thankful to BELSPO

\section{REFERENCES}

[1] Cook, L., "Off-axis three-mirror used off-axis in aperture and field", Proc. SPIE 0183, 207-211 (1979).

[2] Versluys, J., Kendall, D., Moelans, W., Mollet, D., Holbrouck, P., Vrancken, D., Taccola, M., François, M. "The Vegetation instrument: a small scale high performance earth observation instrument", 4S Symposium, (June 2012)

[3] Huby, G., Kleihorst, R., Mellab, K., Grignard, L., "ProbaV, a vegetation satellite", Proc. SPIE 7826, Sensors, Systems, and Next-Generation Satellites XIV, 78260R (October 13, 2010). 
[4] Lencioni, D., Hearn, D., Digenis, C., Mendenhall, J., Bicknell, W., "The EO-1 Advanced Land Imager: An Overview", Lincoln Laboratory Journal 15 (2), (2005).

[5] Defourny, P., Bontemps, S., d'Andrimont, R., Lamarche, C., Moreau, I., Radoux, J., Verhegghen, A., "Global land surface monitoring by medium resolution satellite time series: development and production of several global products", PROBING VEGETATION Conference: from past to future, http://probing.vegetation.be/sites/default/files/pdf/dag1/1630-Pierre\%20DefournyProbing\%20VGT\%20Conference_Defourny_040713_f.pdf

[6] Rodgers, J.,"Control of packaging constraints in optimization of unobscured reflective systems", SPIE, Volume 751 Reflective Optics, 143-149 (1987). 